Delft University of Technology

\title{
Time-varying perceived motion mismatch due to motion scaling in curve driving simulation
}

van Leeuwen, T. D.; Cleij, D.; Pool, D. M.; Mulder, M.; Bülthoff, H. H.

DOI

10.1016/j.trf.2018.05.022

Publication date

2019

Document Version

Accepted author manuscript

Published in

Transportation Research Part F: Traffic Psychology and Behaviour

\section{Citation (APA)}

van Leeuwen, T. D., Cleij, D., Pool, D. M., Mulder, M., \& Bülthoff, H. H. (2019). Time-varying perceived motion mismatch due to motion scaling in curve driving simulation. Transportation Research Part F: Traffic Psychology and Behaviour, 61, 84-92. https://doi.org/10.1016/j.trf.2018.05.022

\section{Important note}

To cite this publication, please use the final published version (if applicable).

Please check the document version above.

\section{Copyright}

Other than for strictly personal use, it is not permitted to download, forward or distribute the text or part of it, without the consent of the author(s) and/or copyright holder(s), unless the work is under an open content license such as Creative Commons.

\section{Takedown policy}

Please contact us and provide details if you believe this document breaches copyrights.

We will remove access to the work immediately and investigate your claim. 


\title{
Time-varying perceived motion mismatch due to motion scaling in curve driving simulation
}

\author{
T.D. van Leeuwen ${ }^{1}$, D. Cleij ${ }^{1,2}$, D.M. Pool ${ }^{1}$, M. Mulder ${ }^{1}$ and H.H. Bülthoff ${ }^{2}$
}

(1) Delft University of Technology, faculty of Aerospace Engineering, Control \& Simulation section, 2629 HS Delft, e-mail : \{d.m.pool, m.mulder\}@tudelft.nl

(2) Max Planck Institute for Biological Cybernetics, Motion Perception and Simulation Group, 72076 Tübingen, e-mail : \{diane.cleij, heinrich.buelthoff\}@tuebingen.mpg.de

\begin{abstract}
In motion simulation, motion input scaling is often applied to deal with the limited motion envelopes of motion simulators. In this research, the time-varying effects of scaling the lateral specific force up or down during passive curve driving in a car driving simulation are investigated through a simulator experiment. It is concluded that lateral specific force scaling has a time-varying effect on the perceived fidelity of a curve-driving simulation. In particular, motion scaling during a curve entry is found to be less detrimental than motion scaling during a curve's sustained part and during the curve exit.
\end{abstract}

Keywords: motion simulation, driving simulators, curve driving, continuous subjective rating

\section{Introduction}

Motion simulators aim to present subjects with a sensation of motion similar to the sensation one would perceive when operating a real vehicle. This is done by combining visual, vestibular, auditory and somatosensory cues. Due to simulators' limited motion envelopes, a conversion from the desired vehicle motion to simulator motion is needed. One of the most straightforward methods for limiting simulator displacement is motion (down)scaling.

Various studies have investigated humans' ability to distinguish motion with different scaling factors [CG10, CG13] and the effect of motion scaling on a simulation's realism [Ber13, Gra03]. These studies conclude that downscaling inertial simulator motion does not necessarily reduce a simulation's fidelity and may even improve it [CG10, CG13, Ber13]. The effects of motion scaling on the control behavior of subjects in a roll and pitch tracking task have also been studied [Ber70, Val12], showing that gains closer to unity improve pilots' control performance.

These studies investigated the effect of motion scaling on the total simulation and did not investigate the time-varying influence of motion scaling. Such an influence is expected since the magnitude of the induced signal distortion over a simulation segment inherently varies over time for typical motion cueing algorithms (such as washout filters [Rei86]). For example, a high-pass filter applied to curve-driving motion may replicate the curve entry well, but filters out the sustained cue in the curve's steady-state part and will induce a false cue at the curve exit [Gra97].

First evidence of a time-varying effect of specific force scaling in longitudinal motion was found by Groen, Valenti Clari and Hosman [Gro01], who concluded that onset cues can be scaled down further than sustained cues in aircraft take-off maneu- vers. Up till now, however, no study has formally verified this finding using continuous subjective evaluations of simulator motion to explicitly measure the effects of motion scaling on perceived simulation fidelity over time.

This paper describes a simulator experiment carried out to investigate the time-varying effects of lateral specific force scaling in curve driving simulation. The method of continuous rating proposed by Cleij et al. [Cle18] is used, in which subjects continuously use a rotary knob in the simulator cabin to indicate their perceived motion mismatch during curve-driving simulation segments.

The structure of this paper is as follows. Following a short formulation of the research goal, the experiment methods are discussed. After this, the experiment results are presented and discussed. The main conclusions resulting from the experiment are drawn at the end of the paper.

\section{Research goal}

The main goal of this research is to investigate whether the effects of lateral specific force scaling on the perceived fidelity of a curve-driving simulation are time-varying. We hypothesized that the effect of lateral specific force scaling on the perceived motion mismatch would not be equal during the curve entry, the sustained part of the curve and the curve exit.

\section{Methods}

To investigate the time-varying effects of motion scaling during motion simulation, a within-subjects simulator experiment was performed. In this experiment subjects were a passenger in a car driving through 
a series of left and right curves. Subjects were instructed to focus on continuously rating their perceived motion mismatch (PMM) using the rating method proposed by Cleij et al. [Cle18].

\section{Participants}

16 Participants (11 male, 5 female) aged between 23 and 40 years old ( $\mu=28.4 \mathrm{yrs}, \sigma=6.1 \mathrm{yrs}$ ) completed the experiment. All were unaware of the goal of the experiment and were (or had been) in possession of a driver's license for at least 3 years $(\mu=9.3$ yrs, $\sigma=4.6 \mathrm{yrs}$ ). On average, the participants drove a car for 3.8 hours per week ( $\sigma=4.4 \mathrm{hrs})$. Six participants were considered novices (first time in a simulator), five were labelled intermediate (some simulator experience) and five were considered experts (researchers on motion cueing).

\section{Apparatus}

The experiment was carried out in the CyberMotion Simulator at the Max Planck Institute for Biological Cybernetics [Teu07], shown in Fig. 1. This eight degree-of-freedom simulator was developed from an industrial robot (Kuka GmbH, Germany). The software package CarSim (Mechanical Simulation Corporation) was used to generate the experiment's visual images and inertial car motion. A screenshot of the outside visual image for subjects during a curve is shown in Fig. 2. The participants indicated their perceived motion mismatch using the rotary knob shown in Fig. 3. Subjects received feedback on their rating on the vehicle dashboard, as shown in Fig. 2.

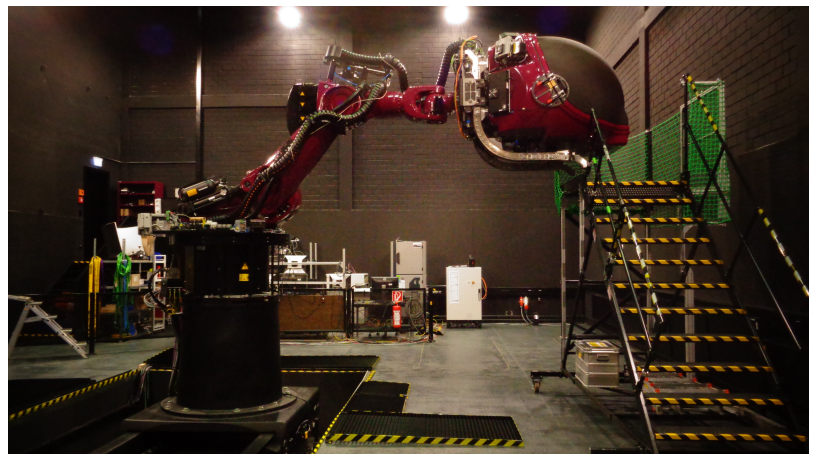

Figure 1: The CyberMotion Simulator (CMS) at the Max Planck Institute for Biological Cybernetics.

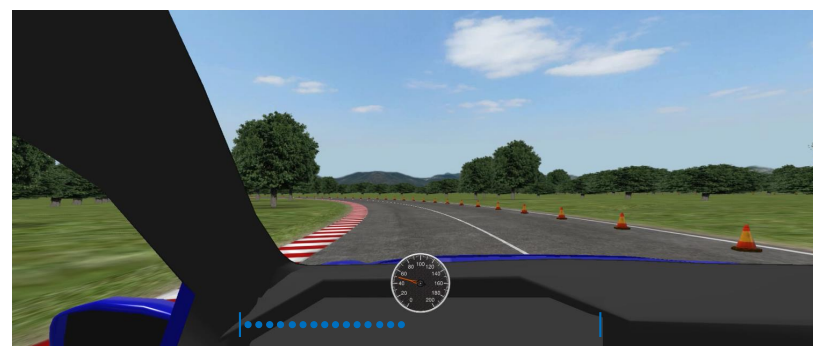

Figure 2: Outside visuals shown during the experiment, showing the white reference line drawn on the road center and the rating bar at the bottom in blue.

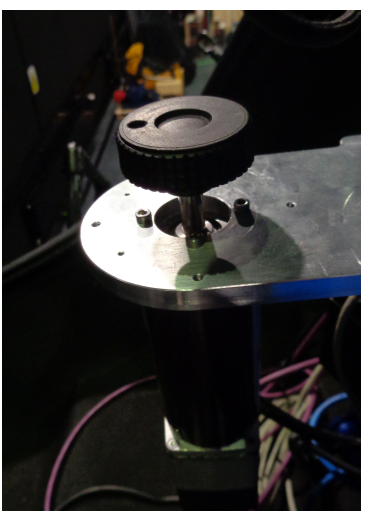

Figure 3: Rotary force-feedback knob (SensoDrive, Germany) used by subjects to indicate their PMM.

The outside visuals were projected on the inside of the simulator cabin, by means of two projectors with an update rate of $60 \mathrm{~Hz}$. The field-of-view of the visual system is approximately 140 deg horizontally and 70 deg vertically [Nie13]. The participants' eye location is approximately $50 \mathrm{~cm}$ below the top of the screen and $150 \mathrm{~cm}$ away from its bottom edge. The latency of the visual cues generated with this system is known to be around $40 \mathrm{~ms}$ [Dro13].

\section{Scenario \& Conditions}

During the experiment runs, a simulated car accelerated from 0 to $50 \mathrm{~km} / \mathrm{h}$, then went through left and right 90-degree turns ( $r=120 \mathrm{~m}$ constant radius) with $150 \mathrm{~m}$ straight sections in between, and finally decelerated back to standstill. Each run of the experiment took 9 minutes, with 11 left and 11 right turns randomly distributed over each run. The vehicle motion generated by CarSim for a left-hand turn is shown in black in Fig. 4.

To keep the lateral jerk low, the automatic CarSim driver steered into and out of turns relatively early and slowly. This steering behaviour ensured the tiltcoordination roll rate remained below the $3 \mathrm{deg} / \mathrm{s}$ perception threshold (for passive driving) for all conditions [Nes16]. In the absence of a visual steering wheel, a line was drawn on the road to assist participants in judging when the automatic driver steered into and out of corners. On straight road segments this line aligned with the participant's head, during curves the car would deviate from this line (see the white line in the middle of the road in Fig. 2).

To simulate the car drive in the CMS, a classic washout filter was used [Gra97]. Such filters first multiply all input (per motion channel) by a fixed gain $k$. Next, the high-frequency motion (which is simulated by directly translating (for acceleration) or rotating (for rotations) the simulator cabin) is determined by applying a third-order high-pass filter $H_{\mathrm{HP}}$ to the (scaled) input:

$$
H_{\mathrm{HP}}(s)=\frac{s^{2}}{s^{2}+2 \zeta_{\mathrm{HP}} \omega_{n_{\mathrm{HP}}} s+\omega_{n_{\mathrm{HP}}}^{2}} \cdot \frac{s}{s+\omega_{n, 2_{\mathrm{HP}}}}
$$

Here $s$ is the Laplace operator, with $\omega_{n_{\mathrm{HP}}}$ the highpass break frequency in rad/s and $\zeta_{H P}$ the high-pass damping ratio. The second term in (1) ensures additional attenuation at lower frequencies through a sec- 

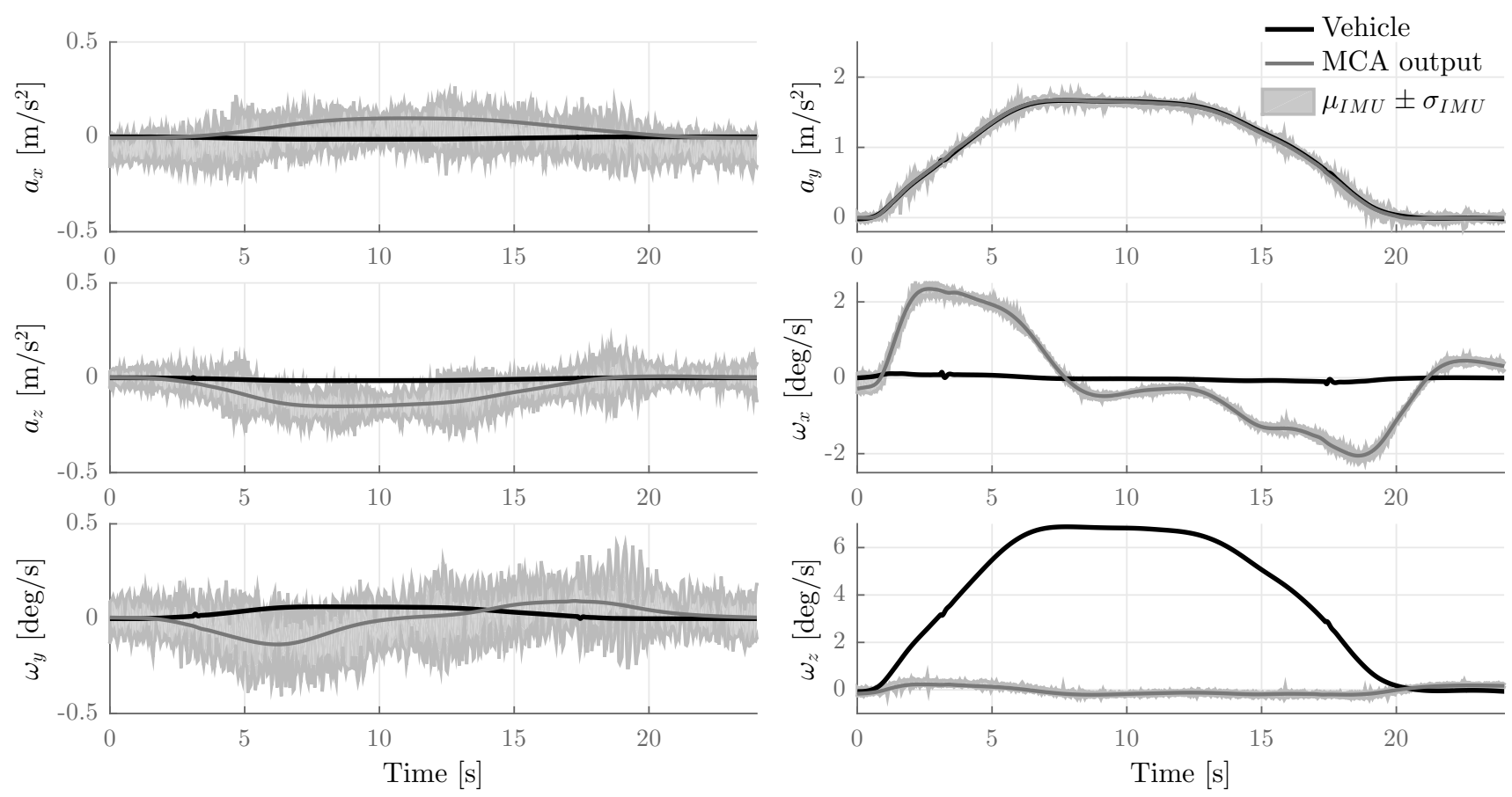

Figure 4: Vehicle (black) and simulator (gray) specific forces and rotational rates during a curve to the left, as recorded in CarSim.

ond break frequency $\omega_{n, 2 \mathrm{Hp}}$, and ensures the simulator returns to its neutral position even for constant specific force inputs. Sustained specific force (which is simulated by applying tilt coordination) is obtained by filtering the (scaled) input using the following lowpass filter $H_{\mathrm{LP}}$ :

$$
H_{\mathrm{LP}}(s)=\frac{\omega_{n_{\mathrm{LP}}}^{2}}{s^{2}+2 \zeta_{\mathrm{LP}} \omega_{n_{\mathrm{LP}}} s+\omega_{n_{\mathrm{LP}}}^{2}}
$$

Here $\omega_{n p p}$ is the low-pass break frequency in rad/s and $\zeta_{\mathrm{LP}}$ is the low-pass damping ratio. For the lateral specific force, these high-pass and low-pass filters were complemented by a band-pass filter, which commanded the simulator's linear rail (which was used to provide additional lateral acceleration) and was defined as:

$$
H_{\mathrm{BP}}(s)=1-H_{\mathrm{HP}}(s)-H_{\mathrm{LP}}(s)
$$

The combination of these three filters ensured that the lateral specific force was effectively simulated one-to-one. During the experiment the parameter settings shown in Table 1 were used. For the condition with $k_{y}=1$, the comparison between vehicle's inertial motion (in black), the washout filter output (in grey) and simulator's inertial motion (measured at $250 \mathrm{~Hz}$ by a Stim $300 \mathrm{IMU}$ positioned some $20 \mathrm{~cm}$ below a subject's eyes, $20 \mathrm{~cm}$ behind the head and $30 \mathrm{~cm}$ to the right) is shown in Fig. 4. The area shaded in grey shows the average IMU measurement after removing bias plus or minus one standard deviation, based on between 23 and 35 samples (depending on the availability of data).

Out of the twenty-two curves in a single run, two featured a one-to-one replication of the lateral specific force (i.e. $k_{y}=1$ ). In the remaining 20 curves, different errors in the lateral specific force cueing were induced. In this paper, only a selection of the experiment data is discussed, namely the six curves featuring a lateral specific force that was purely scaled

\begin{tabular}{|c|c|c|c|c|c|c|}
\hline $\begin{array}{l}\text { Motion } \\
\text { Channel }\end{array}$ & $\begin{array}{l}k \\
{[-]}\end{array}$ & $\begin{array}{l}\omega_{n_{\mathrm{HP}}} \\
{[\mathrm{rad} / \mathrm{s}]}\end{array}$ & $\begin{array}{c}\zeta_{\mathrm{HP}} \\
{[-]}\end{array}$ & $\begin{array}{c}\omega_{n, 2 \mathrm{HP}} \\
{[\mathrm{rad} / \mathrm{s}]}\end{array}$ & $\begin{array}{l}\omega_{n_{\mathrm{LP}}} \\
{[\mathrm{rad} / \mathrm{s}]}\end{array}$ & $\begin{array}{l}\zeta_{\mathrm{LP}} \\
{[-]}\end{array}$ \\
\hline$a_{x}$ & 0.3 & 1.5 & 1 & 0.8 & 1.4 & 1 \\
\hline$a_{y}$ & $k_{y}$ & - & - & - & - & - \\
\hline$a_{z}$ & 1 & 0.5 & 1 & 0.3 & 0 & - \\
\hline$\omega_{x}$ & 0.5 & 0 & - & 0.5 & 0 & - \\
\hline$\omega_{y}$ & 0.5 & 0.5 & 1 & - & 0 & - \\
\hline$\omega_{z}$ & 0.3 & 0 & - & 0.5 & 0 & - \\
\hline
\end{tabular}

Table 1: Washout filter parameter settings

down or up through $k_{y}$. All conditions were spread randomly over each of the four experimental runs performed. An overview of relevant conditions and their curve number and orientation is given in Table 2.

Table 2: Experiment conditions, with their corner number and orientation in each experiment run.

\begin{tabular}{cccccc}
\hline Condition & $k_{y}[-]$ & \multicolumn{4}{c}{ Experiment run } \\
& & E1 & E2 & E3 & E4 \\
\hline $\mathrm{C} 1$ & 1 & $18, \mathrm{R}$ & $13, \mathrm{~L}$ & $14, \mathrm{R}$ & $10, \mathrm{~L}$ \\
\hline $\mathrm{C} 2$ & 1 & $13, \mathrm{~L}$ & $2, \mathrm{R}$ & $22, \mathrm{R}$ & $5, \mathrm{~L}$ \\
\hline $\mathrm{C} 3$ & 0.4 & $2, \mathrm{R}$ & $20, \mathrm{~L}$ & $5, \mathrm{~L}$ & $14, \mathrm{R}$ \\
\hline $\mathrm{C} 4$ & 0.5 & $16, \mathrm{R}$ & $6, \mathrm{~L}$ & $11, \mathrm{R}$ & $2, \mathrm{~L}$ \\
\hline $\mathrm{C} 5$ & 0.6 & $20, \mathrm{~L}$ & $10, \mathrm{R}$ & $9, \mathrm{R}$ & $7, \mathrm{~L}$ \\
\hline $\mathrm{C} 6$ & 0.8 & $10, \mathrm{~L}$ & $1, \mathrm{R}$ & $21, \mathrm{~L}$ & $16, \mathrm{R}$ \\
\hline $\mathrm{C} 7$ & 1.2 & $6, \mathrm{~L}$ & $17, \mathrm{~L}$ & $17, \mathrm{R}$ & $20, \mathrm{R}$ \\
\hline $\mathrm{C} 8$ & 1.275 & $21, \mathrm{R}$ & $12, \mathrm{R}$ & $1, \mathrm{~L}$ & $12, \mathrm{~L}$ \\
\hline
\end{tabular}

\section{Independent variables \& Dependent measures}

The specific lateral force conditions listed in Table 2 serve as the independent variable of this experiment. Conditions C1 and C2 serve as the experiment baseline and allow for checking whether subjects are 


\begin{tabular}{|c|}
\hline \\
$\begin{array}{c}\text { Zero } \\
\text { mismatch }\end{array}$
\end{tabular}

Figure 5: Rating bar displayed to subjects.

able to give consistent ratings. Conditions $\mathrm{C} 3-\mathrm{C} 6$ investigate the effect of downscaling the lateral specific force, conditions C7 and C8 investigate the effect of upscaling the lateral specific force. The gain of condition C8 was chosen such that the maximum tilt-coordination roll rate remained just below $3 \mathrm{deg} / \mathrm{s}$.

The continuous ratings given by subjects are the sole dependent measure. Subjects gave this rating by turning the knob shown in Fig. 3, which had a range of -60 to 60 degrees. This indicated knob angle was converted to a percentage rating through:

$$
\text { Rating }=\frac{\text { Rating knob angle }+60^{\circ}}{120^{\circ}} \cdot 100 \%
$$

Subjects were shown their current rating through a bar on their screen, see Figs. 2 and 5.

\section{Experiment procedure}

Before the experiment subjects were briefed through an instruction form, accompanied by additional verbal explanation. Subjects were made aware of the fact that the inertial motion they felt would not always correspond to the motion they saw during the experiment, and that it was their task to continuously rate the discrepancy between these two by turning the rotary knob. Subjects were instructed to rate this perceived motion mismatch on the scale shown in Fig. 5, which ranged from zero (no mismatch between the visual and inertial motion) to a maximum rating (for the biggest mismatch during the experiment). Examples of visual-motion mismatches were explained to subjects.

After these instructions, the participants signed an informed consent form and filled in a simulator sickness questionnaire. The latter form was repeated at the end of the experiment to verify whether simulator sickness could have influenced the results of this study. Two subjects reported minor simulator sickness symptoms, however, these (experienced) subjects indicated that these symptoms did not influence their ratings. Hence, all data $(n=16)$ were used.

At the start of the experiment, subjects performed a familiarization run consisting of ten curves of condition $\mathrm{C} 1$. The goal of this run was to let subjects get used to the driving style of the automatic CarSim driver, which steered into and out of curves relatively early to reduce lateral jerk and thus keep the tiltcoordination roll rate below $3 \mathrm{deg} / \mathrm{s}$ in all conditions. After this run, subjects were instructed to take this run as a reference for coherent motion.

Next, subjects were presented two training runs. These runs featured 22 corners with the conditions thought to cause the smallest and largest PMM. During the first training run, subjects were asked to focus on what motion corresponded most and least to the motion they saw, allowing them to determine the end points of their rating scale (see Fig. 5). Following this training run, subjects were specifically asked to describe the motion that felt worst.

During the second training run, subjects were asked to continuously provide ratings of their PMM on the scale developed during the first training run. Subjects were instructed they could change their rating scale during this second training run (should they encounter motion that was even worse than previously experienced), but were instructed to keep a constant rating scale from the training onwards. After going through the experiment training phase, the four experimental runs (E1-E4) were presented in random order.

To prevent fatigue from affecting the experiment's results a break outside the simulator was introduced after the first block of four runs (consisting of the familiarization run, both training runs and the first experiment run). Following this break the familiarization run was repeated, followed by the final three experiment runs. Between runs within a block, subjects had the possibility to take a break in the stationary simulator. In total, the experiment lasted just under two hours (1.25 hours of simulator time).

\section{Hypotheses}

Prior to the experiment, the following hypotheses were formulated:

H1 The lateral specific force can decrease by $50 \%$ during the sustained part of a curve without a significant increase in PMM [CG13].

H2 The lateral specific force can increase by $30 \%$ during the sustained part of a curve without a significant increase in PMM [CG13].

H3 The effect of lateral specific force scaling on the perceived motion mismatch will not be equal during the curve entry, the steady-state part of the curve and the curve exit [Gro01].

\section{Results}

For all participants, the ratings given over the four experiment runs were averaged (one subject indicated to have misunderstood the experiment instructions after the first run, for this subject only the final three runs were averaged). The means and spread of these 16 average ratings are shown in Fig. 6, where one can see that subjects returned their rating to (almost) $0 \%$ on straight sections (at $t=0 s$ and $t>20 s$ ), indicating ratings are not influenced by the rating of the previous condition. From Fig. 6 one can also conclude that PMM ratings increase for decreasing $k_{y}$ from $k_{y}=1$ onwards, matching the findings of similar studies by Jamson [Jam10] and Berger [Ber10].

The time-varying effect of motion scaling was studied by analyzing the curve entry, the sustained part of the curve and the curve exit separately. Based on the lateral specific force profile shown in Fig. 6, onset was defined as $t=1-5 \mathrm{~s}$, the sustained curve was defined as $t=8-14 \mathrm{~s}$ and the curve exit as $t=16-23 \mathrm{~s}$. Intermediate sections of time were ignored, in order to clearly distinguish between the curve's onset, steady part and exit.

Since subjects on average showed a rating delay of $1 s$, the analysed rating windows were moved one 
second compared to the time windows given previously. For all analyses non-parametric statistical tests were chosen at a Sidak-corrected significance level of $\alpha=0.05$. When comparing these results to those of other studies, it should be noted that our subjects had been calibrated to the unity gain condition during the familiarization phase.

Curve entry Boxplots of the average subject ratings between $t=2 s$ and $t=6 s$ are shown in Fig. 7a. From Fig. 7a one can see that downscaled conditions are rated slightly higher on average, and generally have a spread similar to that of conditions $\mathrm{C} 1$ and C2. Also, the upscaled conditions show a slightly higher average rating and an increase in spread in ratings compared to the one-to-one conditions. One can also see that conditions $\mathrm{C} 1$ and $\mathrm{C} 2$ were rated equal, hence only $\mathrm{C} 1$ was used for comparing scaled conditions to the unscaled version.

A Friedman test performed on these average ratings showed a significant overall effect $\left(\chi^{2}(6)=\right.$ $13.6, p=0.034)$ to be present. Six Sidak-corrected post-hoc Wilcoxon signed-rank tests showed that only the ratings for condition C3 $\left(k_{y}=0.4, p=\right.$ $0.005)$ differed significantly from those of the oneto-one condition.

Sustained curve To obtain a rating for the sustained part of the curve ratings between $t=9 \mathrm{~s}$ and $t=15 \mathrm{~s}$ were averaged, the results of which are shown in Fig. 7b. In Fig. 7b one can observe the downscaled conditions to be rated higher as the scaling factor $k_{y}$ decreases. Conditions C7 and C8 have a mean rating similar to $\mathrm{C} 1$ and $\mathrm{C} 2$, however the spread has increased. Since conditions $\mathrm{C} 1$ and $\mathrm{C} 2$ are again rated equal, only $\mathrm{C} 1$ ratings were used for comparison.

A Friedman test of the remaining seven distributions showed a significant overall effect $\left(\chi^{2}(6)=\right.$ $51.7, p<0.001)$ between the seven conditions. Six Sidak-corrected post-hoc Wilcoxon signedrank tests showed that ratings for conditions $\mathrm{C} 3$,
C4 and C5 differed significantly from those of condition $\mathrm{C} 1(p=0.001)$.

Curve exit For the curve exit the average ratings between $t=17 \mathrm{~s}$ and $t=24 \mathrm{~s}$ were analyzed, resulting in the rating distributions shown in Fig. 7c. Similar to the sustained part of the curve, ratings increase as the scaling factor decreases. Also, the conditions for which $k_{y}>1$ appear to be rated similar to the one-to-one conditions. Condition C2 was again omitted in the comparisons, since it is nearly equal to condition $\mathrm{C} 1$.

A Friedman test showed a significant overall effect $\left(\chi^{2}(6)=34.9, p<0.001\right)$ to be present in these seven conditions. Six Sidak-corrected post-hoc Wilcoxon signed-rank tests showed that the ratings of conditions C3, C4 and C5 differed significantly from those of condition $\mathrm{C} 1(p<0.005)$.

Based on the results obtained during the sustained part of the curve, hypothesis $\mathrm{H} 1$ is rejected. Instead it is concluded that subjects report a significant increase in PMM in case the lateral specific force is scaled down by $40 \%$ or more (as the ratings of conditions C3, C4 and C5 differed significantly from those of condition $\mathrm{C} 1$ ). Based on these results, a minimal scaling factor $k_{y}$ of 0.7 would be more appropriate. Hypothesis $\mathrm{H} 2$ can not be rejected, as none of the two conditions with a gain larger than unity was rated to be "too strong".

Based on the obtained results, hypothesis $\mathrm{H} 3$ is not rejected, as it has been shown that the lateral specific force can be scaled down further during the curve entry (Fig. 7a), than during the remaining part of the curve (Fig. 7b-c).

\section{Discussion}

In this paper, an experiment to study the time-varying effect of lateral specific force scaling during curve driving was presented. Based on the ratings shown in

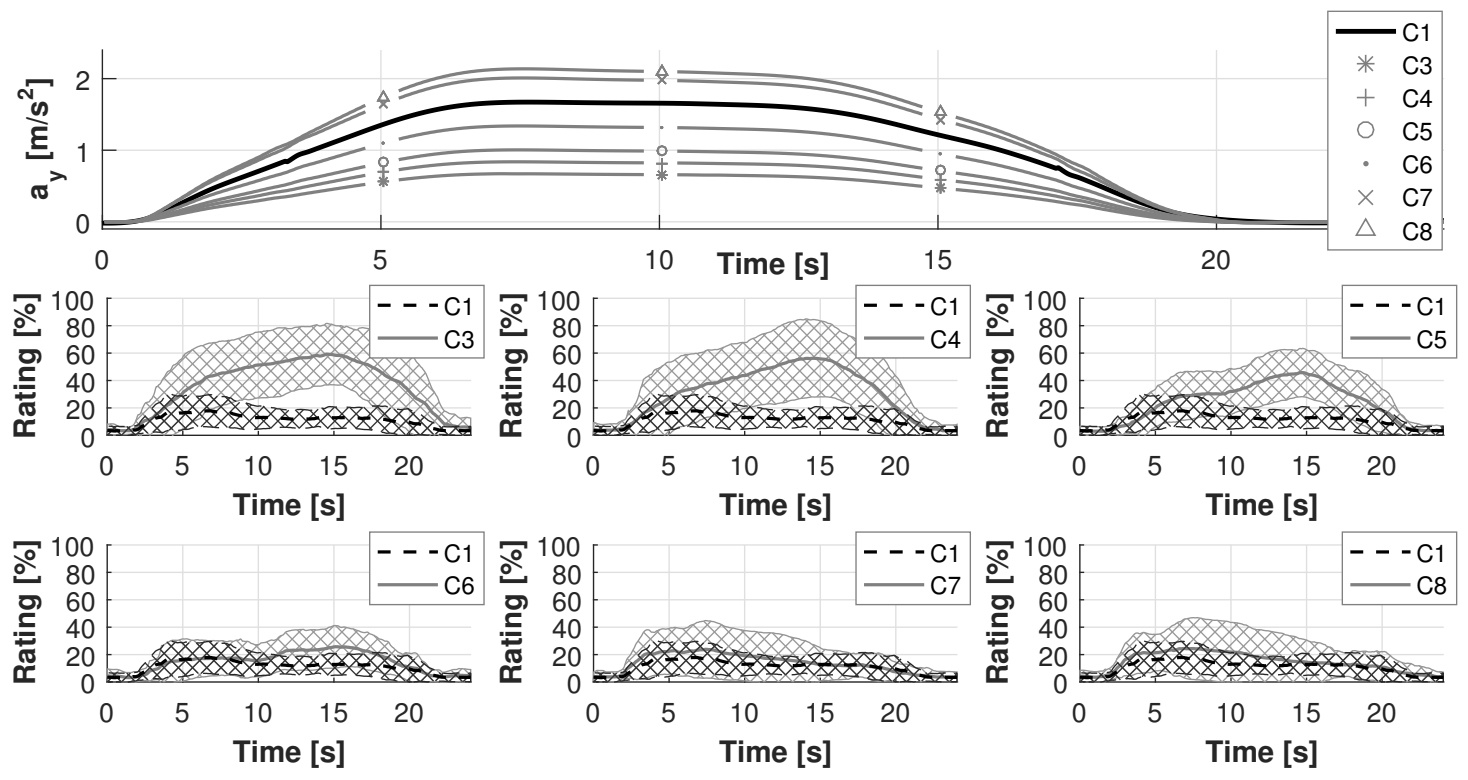

Figure 6: Lateral specific force presented in each condition (top), and average ratings given for all conditions. Shaded areas indicate the mean rating plus/minus one standard deviation. 


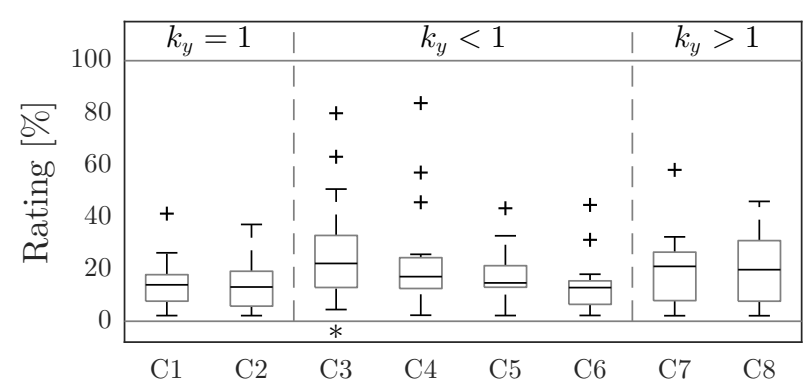

(a) Boxplot of average ratings for the curve entry.

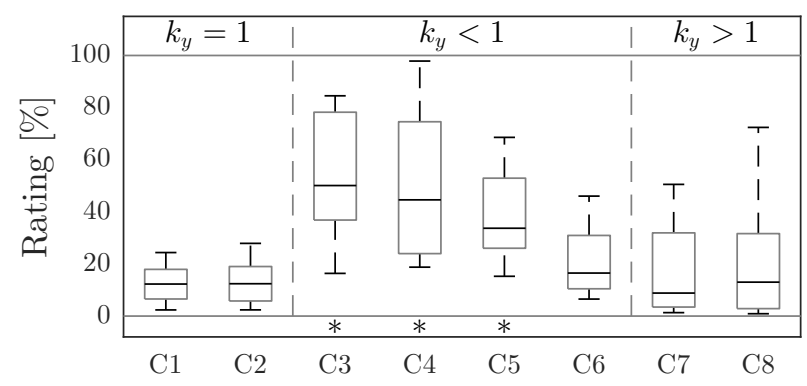

(b) Boxplot of average ratings for the sustained curve.

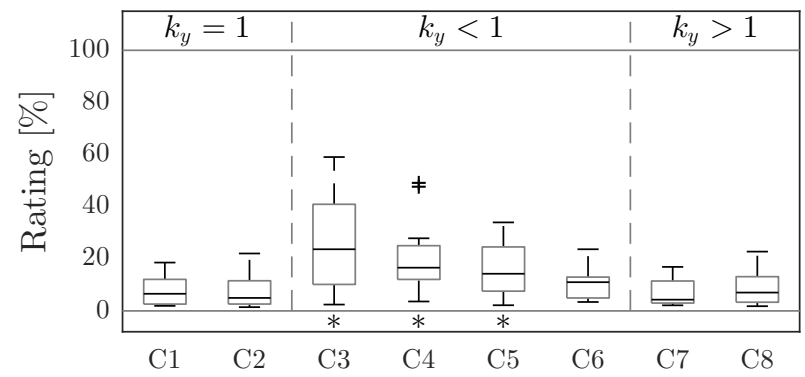

(c) Boxplot of average ratings for the curve exit.

Figure 7: Rating distributions for three curve segments. Stars indicate significant differences w.r.t. condition $\mathrm{C} 1$.

Fig. 6, it was concluded that the lateral specific force can be scaled down by $30 \%$ during the sustained part of a curve, without subjects indicating a mismatch between their visual and inertial motion. No significant difference in perceived motion mismatch (PMM) was found in case the lateral specific force was increased by up to $27.5 \%$. This may however be caused by the limited number of subjects. Also, it was concluded that the lateral specific force can be scaled down further during the onset of a curve, as compared to the remainder of the curve.

These conclusions are based on continuous ratings given by subjects throughout their runs to indicate their PMM. These ratings, though subjective, showed that all subjects consistently provide their maximum rating (of $100 \%$ ) for the same condition (one of the 14 conditions not discussed in this paper). While this allowed for anchoring the ratings of different subjects, this still implies that a rating increase from $40 \%$ to $60 \%$ might not correspond to an equally large increase in PMM. Analysis of the relative magnitude of the ratings given by each subject is, however, due to the repeated-measures setup of our experiment, still valid. Due to the different use of the rating scale per subject, the overall rating data were in general also not normally distributed and hence analyzed using non-parametric statistical tests.

It is noted that subjects in this experiment had a passive role, whereas in most simulation environments subjects have an active task. This could mean that in most simulation applications the values found for the allowable up- and downscaling factors might be even further away from 1.0, since participants may notice motion mismatches less during an active task [Nes12]. On the contrary, subjects may better notice a motion mismatch during an active task since they are better aware of what motion their input should result in. Further experiments could investigate which of the two effects mentioned above is stronger.

The values for both the allowable up- and downscaling factor are slightly higher than the factors found by Correia Grácio et al. [CG13]. This indicates that in our research, we have found a coherence zone (the region of allowable scaling factors $k$ for which the inertial motion feels coherent with the visual motion) which is shifted upwards compared to the one found by Correia Grácio et al. in their research. This finding is attributed to the fact that, unlike in Correia Grácio's research, subjects were given the condition with a unity gain as a reference in our experiment. Additionally, the simulators used in both experiments produced different types of motion, and may have differed in terms of motion quality (noise, delay, etc.).

The values found for the allowable up- and downscaling factors are valid for the lateral specific force in a low-frequency and smooth curve driving scenario. Separate research would be needed to comment on suitable values for these factors in higher frequency cornering motion, such as slalom driving, or non-smooth driving conditions, for example when driving over bumps. Also, further research would be needed to determine the allowable scaling factors for other motion channels (longitudinal and vertical specific force, as well as rotations).

The fact that the lateral specific force can be scaled down further during the curve entry might be explained by the accompanying reduction in tilt coordination roll rate, as shown in Fig. 8. As can be seen the tilt coordination roll rate for the unity gain condition $\mathrm{C} 1$ is around $2.5 \mathrm{deg} / \mathrm{s}$. Even though this rate is below the theoretical threshold value of $3 \mathrm{deg} / \mathrm{s}$ for passive activities, some subjects still reported feeling the tilt. In case the lateral specific force is scaled down subjects will perceive the motion to be too weak, but will also experience a lower roll rate. It is hypothesized that these effects may, to some extent, cancel each other, leading to similar subjective ratings during the curve entry (see Fig. 6).

Besides this false roll rate, four factors can be distinguished which could have influenced this study's results. The first of these concerns the yaw rate experienced by subjects : although the lateral specific force was simulated one-to-one, the yaw rate $\omega_{z}$ was not. Because the gain for $\omega_{z}$ in the high-pass filter was set to 0.3 , it could be that subjects deemed a scaled-down lateral specific force more realistic because it better matched to the smaller yaw rate onset.

Secondly, the three segments distinguished in each curve (onset, sustained curve and exit) were not of equal length, owing to the fact that the sustained part of the curve had to be long enough to simulate all 21 different conditions in the full experiment. This diffe- 


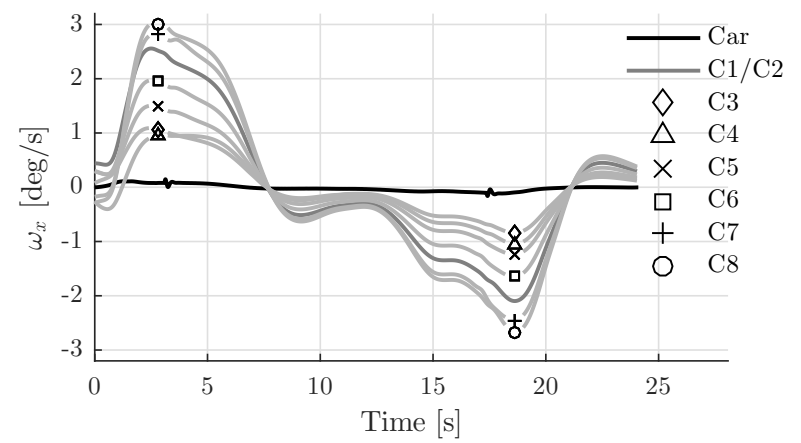

Figure 8: Tilt coordination roll rates during the experimental conditions.

rence in segment length could have resulted in subjects rating the onset, sustained part and exit differently as they felt the length of the segment wasn't realistic, possibly resulting in the differences found between segments.

In our analysis, not all recorded rating data were used. We focused on three specific segments - curve entry ( $2-6 s$ after start of the curve), sustained curve $(9-15 s)$, and curve exit $(17-24 s)-$ of data to assess subjects' ratings of each curve segment. The choice of these segments was based on the lateral specific force profile and the observed delay in subjects' ratings and made such that there was no overlap between segments. A different choice of time segments, however, could have resulted in different statistical differences being found. Shorter time segments could increase the probability of finding a significant difference by chance, longer time segments could hide more local effects (especially for the curve entry and exit) if they include the time segment where subjects still are/have returned to neutral ratings.

A final potential factor concerns the quality of the visual motion presented by the simulator. Because of the CyberMotion Simulator's cabin structure, the visual projection is done on a double curved screen by two separate projectors. The limited focal length of, and overlap between, these projectors could have resulted in differences between the visual images a subject saw during the experiment and would see in reality, possibly influencing the ratings subjects gave.

\section{Conclusion}

In this paper the time-varying effect of lateral specific force scaling during a curve driving simulation was investigated. Through a simulator experiment it was shown that such a time-varying effect is clearly present. Specifically, the lateral specific force in curves can be scaled down further during the onset of a curve, than during the sustained part of the curve and during the curve exit. Also, it was shown that for low-frequency curve driving maneuvers the lateral specific force can be scaled down by $30 \%$, and up by at least $30 \%$. The results of this study can be used to improve simulator performance in the future, by scaling down motion where possible. Any part of the simulator motion envelope that is saved using this approach can then be used to simulate the inertial motion more realistically.

\section{References}

$\mathrm{H}$. Bergeron, Investigation of Motion Requirements in Compensatory Control Tasks, IEEE Transactions on Man-Machine Systems, vol. 11(2) : 123-125, 1970.

D. Berger, J. Schulte-Pelkum and H. Bülthoff, Simulating believable forward accelerations on a Stewart motion platform, ACM Transactions on Applied Perception, vol. 7(1), 2010.

A. Berthoz, W. Bles, H. Bülthoff, B. Correia Grácio, P. Feenstra, N. Filliard, R. Hühne, A. Kemeny, M. Mayrhofer, M. Mulder, H. Nusseck, P. Pretto, G. Reymond, R. Schlüsselberger, J. Schwandtner, H. Teufel, B. Vailleau, M. van Paassen, M. Vidal and M. Wentink, Motion Scaling for High-Performance Driving Simulators, IEEE Transactions on Human-Machine Systems, vol. 43(3) : 265276, 2013.

B. Correia Grácio, M. van Paassen, M. Mulder and M. Wentink, Tuning of the lateral specific force gain based on human motion perception in the Desdemona simulator, in AIAA Guidance, Navigation, and Control Conference, Toronto, Ontario, 2010.

B. Correia Grácio, A. Valente Pais, M. van Paassen, M. Mulder, L. Kelly and J. Houck, Optimal and Coherence Zone Comparison Within and Between Flight Simulators, Journal of Aircraft, vol. 50(2) : 493-507, 2013.

D. Cleij, J. Venrooij, P. Pretto, D. M. Pool, M. Mulder and H. H. Bülthoff, Continuous Subjective Rating of Perceived Motion Incoherence During Driving Simulation, IEEE Transactions on Human-Machine Systems, vol. 48(1) : 17-29, 2018.

F. M. Drop, D. M. Pool, M. M. van Paassen, M. Mulder and H. H. Bülthoff, Feedforward and Feedback Control Behavior in Helicopter Pilots during a Lateral Reposition Task, in AHS 69th Annual Forum, 2013.

P. Grant and L. Reid, Motion Washout Filter Tuning : Rules and Requirements, Journal of Aircraft, vol. 34(2) : 145-151, 1997.

P. Grant, M. Blommer, B. Artz and J. Greenberg, Analysing classes of motion drive algorithms based on paired comparison techniques, in Proceedings of the Driving Simulation Conference (DSC) North America, Dearborn, USA, Oct. 8-10, 2003, University of Toronto \& Ford Motor Company, 2003.

E. Groen, M. Valenti Clari and R. Hosman, Evaluation of Perceived Motion During a Simulated Takeoff Run, Journal of Aircraft, vol. 38(4) : 600-606, 2001.

A. Jamson, Motion Cueing in Driving Simulators for Research Applications, Phd thesis, The University of Leeds - Institute for Transport Studies, United Kingdom, 2010.

A. Nesti, C. Masone, M. Barnett-Cowan, R. Robuffo Giordano, $\mathrm{H}$. Bülthoff and P. Pretto, Roll rate thresholds and perceived realism in driving simulation, in DSC 2012 Europe : Driving Simulation Conference \& Exhibition, Paris, France, Sep. 6-7, 2012, Max Planck Institute for Biological Cybernetics, 2012.

A. Nesti, S. Nooij, M. Losert, H. H. Bülthoff and P. Pretto, Roll rate perceptual thresholds in active and passive curve driving simulation, Simulation, vol. 92(5) : 417-426, 2016.

F. M. Nieuwenhuizen and H. H. Bülthoff, The MPI CyberMotion Simulator : A Novel Research Platform to Investigate Human Control Behavior, Journal of Computing Science and Engineering, vol. 7(2) : 122-131, 2013.

L. Reid and M. Nahon, Flight Simulation Motion-Base Drive Algorithms : Part 1 - Developing and Testing the Equations, UTIAS report, no. 296, University of Toronto Institute for Aerospace Studies, 1986.

H. Teufel, H.-G. Nusseck, K. Beykirch, J. Butler, M. Berger and H. Bülthoff, MPI Motion Simulator : Development and Analysis of a Novel Motion Simulator, in Proceedings of the AIAA Modeling and Simulation Technologies Conference and Exhibit, Hilton Head, South Carolina, Aug. 20-23, 2007, American Institute of Aeronautics and Astronautics, 2007.

A. R. Valente Pais, D. M. Pool, A. De Vroome, M. M. Van Paassen and M. Mulder, Pitch Motion Perception Thresholds During Passive and Active Tasks, Journal of Guidance, Control \& Dynamics, vol. 35(3) : 904-918, 2012. 